\title{
Case Report on a 21 Years Old Female Patient with Acute Pancreatitis in Cystic Fibrosis
}

\author{
Carmen Fierbinteanu Braticevici ${ }^{1 *}$, Ana Cristina Hodina ${ }^{1}$, Gabriela Oprea $^{1}$ and Mariana Jinga ${ }^{2}$ \\ ${ }^{1}$ Carol Davila University of Medicine and Pharmacy, Medical Clinic II and Gastroenterology, University Hospital Bucharest, Bucharest \\ ${ }^{2}$ Carol Davila University of Medicine and Pharmacy, Internal Medicine and Gastroenterology Clinic, Central University Emergency Military Hospital, Bucharest, Romania
}

Received: December 10, 2013; Accepted: June 23, 2014; Published: June 25, 2014

*Corresponding author: Carmen Fierbinteanu Braticevici, Medical Clinic II and Gastroenterology, University Hospital Bucharest, Tel: + 40744206268, E-mail address: cfierbinteanu@yahoo.com

\begin{abstract}
Acute pancreatitis is a rare complication in patients with cystic fibrosis. We present a case of a young female with a history of bronchiectasis and Bartter syndrome, who was admitted in our hospital with an acute episode of pancreatitis. We had diagnosed the case as acute pancreatitis, but we did not have a cause for it. She denied drinking alcohol, or consuming drugs. Abdominal trauma was not reported. There was no stone or sludge in the gallbladder and biliary ducts, no malformation on the biliary ducts, and no dyslipidemia. As, the patient had a history of bronchiectasis and Bartter syndrome; we had to look for cystic fibrosis and therefore, we recommended the sweat test and genetic tests. The results obtained for heterozygote $M$ 2183AA>G: 7T, 9T and the sweat test were positive.
\end{abstract}

However, the genetic analyses of her parents could not be performed. We concluded the diagnosis as acute pancreatitis due to cystic fibrosis modifications. The patient was also pancreatic sufficient (the evaluation of exocrine pancreatic function was in normal ranges).

Keywords: Acute Pancreatitis; Cystic Fibrosis; Genetic Marker

\section{Introduction}

Cystic fibrosis (CF) is the most common monogenic disease in people of Caucasian origin. CF is an autosomal recessive, multisystemic disease, with chronic progressive evolution. One in 22 to 25 Caucasians are heterozygotes; this mutation is the most common monogenic mutation among the Caucasians. The average age at diagnosis is 2.9 years. The median survival is 32 years for males and 29 for females. ${ }^{[1]}$ The genetic anomaly is associated with defects in the expression of the gene which codes for the "Cystic Fibrosis Transmembrane Regulator (CFTR)". CFTR is a chloride channel which provides the efficient transfer of ions across epithelial cell membranes. CF is characterized by wide phenotypic variability, resulting in significant clinical differences between patients, in terms of severity and complications.

Acute pancreatitis and chronic pancreatitis describe inflammatory syndromes of the pancreas. Acute pancreatitis is characterized by sudden abdominal pain, elevated serum levels of digestive enzymes ( $>3 \times$ normal) and typical changes on abdominal imaging. ${ }^{[2,3]}$ Two out of the following three criteria should therefore, be present to diagnose AP-characteristic (severe) abdominal pain, serum amylase and/or lipase exceeding 3 times the upper limit of normal, and/or characteristic abdominal imaging findings (strong recommendation, moderate quality of evidence). ${ }^{[4]}$ Chronic pancreatitis is characterized by irreversible damage to the pancreas as a result of inflammation usually characterized by chronic inflammation with variable pain, calcifications, necrosis, fatty replacement, fibrosis, scarring and other complications. ${ }^{[5-8]}$ Both the forms of pancreatitis were once considered to be two distinct diseases; however, it is now assumed that they are usually linked by recurrent acute pancreatitis (as an intermediate step).

\section{Case Report}

A female Caucasian patient aged 21 years, was admitted in our department, with symptoms of abdominal pain, nausea and vomiting; that occurred 24 hours before admission. She was diagnosed with Bartter syndrome and bronchiectasis, at 3 and 16 years of age, respectively.

\section{Physical examination readings were as follows}

Body temperature- $36.5{ }^{\circ} \mathrm{C}$, Body Mass Index (BMI)- $23 \mathrm{~kg}$ $/ \mathrm{m}^{2}$, Pulse- 90 beats per minute, Blood pressure- $110 / 60 \mathrm{~mm}$ of $\mathrm{Hg}$, Respiratory rate- 16 breaths per minute, and Oxygen saturation- 96\% (while she was breathing ambient air). Her abdomen was soft and tender to palpation in epigastric region, with normoactive bowel sounds and no hepatosplenomegaly.

\section{Results of laboratory evaluation included}

Amylase (3xn) and lipase (6xn) levels were higher than normal levels. Levels of total bilirubin, aminotransferases, albumin, glucose, and electrolytes were in the normal range. The white blood cell count, hematocrit count, and the platelet count were also normal. The ultrasound evaluation revealed no abnormalities in the pancreas, cholecyst, and biliary ducts (no stone or sludge on the gallbladder). Reports for Abdominal and pelvic CT (figure 1), Magnetic Resonance Cholangiopancreatography, and Ultrasound Endoscopy were also normal. Chest radiography (figure 2) and CT chest scan showed bilateral bronchiectasis. Spirometry revealed an obstructive dysfunction. 


\section{Treatment}

Treatment was initiated with bowel rest, proton pump inhibitor, painkillers, with improving symptoms. Because of the acute pancreatitis and her history of bronchiectasis, we recommended genetic tests for cystic fibrosis and sweat test. The level of sweat chloride was $78 \mathrm{mmol} / \mathrm{l}$.

The panel mutation analyses performed by The Romanian Genetic Institute included 37 mutations as follows: DF 508, DI507, F508C, I502T, i706del17, 1677delta, G542X, 1717G>A, R553X, Q522X, G551D, S549RA $>$ C, 2183AA $>$ G, NI303K, 4016inst, $3849+10 \mathrm{kbc}>\mathrm{T}, \mathrm{G} 85 \mathrm{E}, 711+5 \mathrm{G}>\mathrm{A}, 578+5 \mathrm{G}>\mathrm{A}, \mathrm{W} 1282 \mathrm{X}, \mathrm{G} 1244 \mathrm{E}$, 621+1G>T, R117H, 852del22, T338I, R347P, i259insa, R1066H LI065P, L1077P, D1152H, 4382dela, R1162X, R1158X, S912X, I148T, 3199del6. The panel mutation testing found that the patient is heterozygote 7T, 9T, 2183AA $>$ G. Another mutant allele, in order for this patient to carry a genetic diagnosis of $\mathrm{CF}$, was not identified. We concluded that the patient is either a carrier with a positive sweat test or with unidentified second mutation (compound heterozygote).

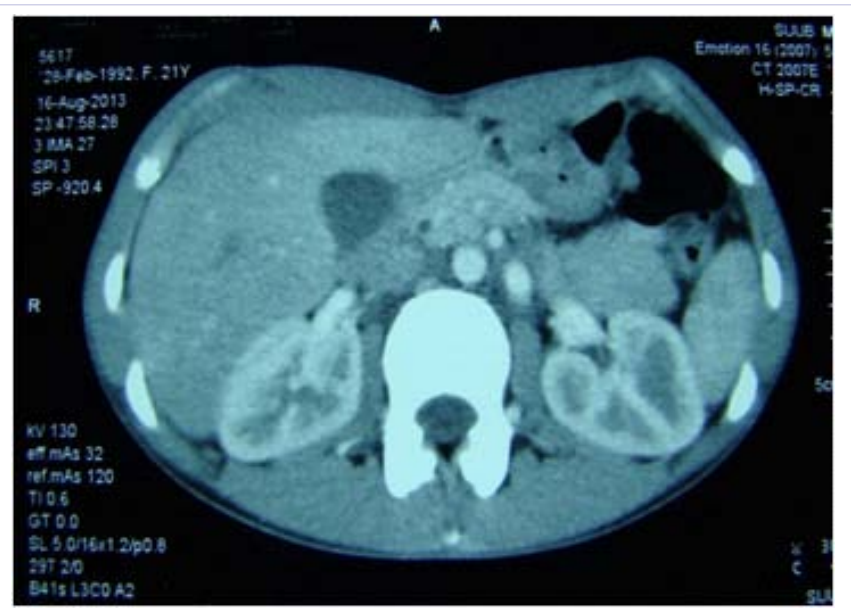

Figure 1: Abdominal CT.

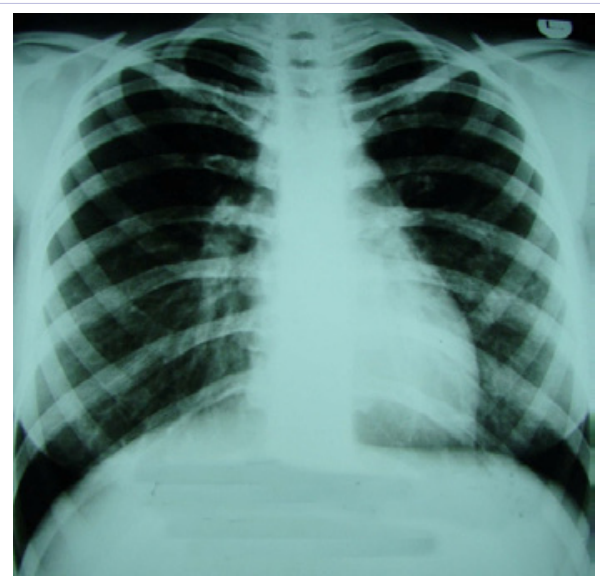

Figure 2: Chest radiography
We considered the diagnosis of acute pancreatitis secondary to $\mathrm{CF}$ based on clinical data, and positive sweat test. Although the genotyping may assist in the diagnosis, it alone cannot rule out the diagnosis of $\mathrm{CF}$. ${ }^{[9]}$

After a period of six months from the recorded episode of acute pancreatitis, the patient had undergone evaluation of exocrine pancreatic function. The 72-hour fecal fat and stool fecal elastase 1 tests were performed. Fat loss was $3.5 \mathrm{~g} / 24$ hours, and the value for fecal elastase 1 was $245 \mu$ g elastase/g, revealing that the patient is pancreatic sufficient.

\section{Discussion}

Usually extra pulmonary manifestations of CF include pancreatic exocrine insufficiency, which is seen in $90 \%$ of patients and leads to fat malabsorption and malnutrition. Acute pancreatitis may develop occasionally. Other gastrointestinal (GI) complications include distal intestinal obstruction syndrome, volvulus, intussusception, and rectal prolapse. CF also affects the endocrine pancreas (diabetes mellitus in approximately $20 \%$ of adults), the hepato-biliary system (fatty liver, cirrhosis, portal hypertension, cholelithiasis, and cholecystitis), the genito-urinary (male infertility and epididymitis), and the skeletal (retardation of growth, osteoarthropathy, osteopenia) systems. Digital clubbing appears in childhood, in virtually all the symptomatic patients. Although fertility may be decreased in women with CF secondary to thickened cervical mucus, many women with $\mathrm{CF}$ have tolerated pregnancy well.

Acute pancreatitis represents a rare manifestation of CF occurring in approximately $2 \%$ of patients; more frequently manifestation is chronic pancreatitis. ${ }^{[10,11]}$ The medical records of 3306 patients with CF from twenty-six centers, showed that only 61 cases of pancreatitis were reported, leading to a prevalence of $1.84 \%$ (95\% CI: $1.39-2.30 \%$ ). The mean age of the patients with pancreatitis was 24.4 years (SD: 10.8 years). Pancreatitis occurs mainly during adolescence and young adulthood. It is more common in patients with pancreatic - sufficient CF (PSCF) (10.3\%), but rarely occurs among patients with pancreatic insufficiency $(0.5 \%)$. ${ }^{[10]}$ However, only $15-20 \%$ of patients with PS-CF develop pancreatitis, suggesting the potential equal contribution of genetic factors (severity of CFTR mutations) and environmental factors. ${ }^{[12]}$

In a large study (till date) of subjects with PS-CF, Ooi et al showed an association between risk of pancreatitis and severity of CFTR genotype. They concluded that the risk of pancreatitis increased in patients with mild CFTR dysfunction as compared with those with moderate-severe CFTR genotype. ${ }^{[13]}$ Conforming to this hypothesis, our patient, who carries a mild mutation on at least one allele (heterozygote $7 \mathrm{~T}, 9 \mathrm{~T}, 2183 \mathrm{AA}>\mathrm{G}$ ), has some residual ion channel function that usually confers sufficient exocrine pancreatic function.

Advances in molecular analysis techniques have led to the identification of $>1600$ CFTR mutations. The first study in Romania regarding CF concluded that the prevalence of CFTR 
mutations were as following: $\Delta \mathrm{F} 508$ was $36,6 \%, 2043$ delg $2 \%$, W1282X 1,7\%, 1717-2A->G, I148T, 621+1G->T, G542X, R553X, G576X, 1898+1G->A, 2183AA->G for each about $1.4 \%{ }^{[14]}$

Leonardi et al., have reported $\Delta \mathrm{F} 508$ as the first recognized mutation of the CFTR gene. The prevalence of this mutation is $2.8 \%$ in Caucasian population. It is also responsible for around $70 \%$ of the typical form of cystic fibrosis in homozygosis. ${ }^{[15]}$

Because the patient did not have genetic test to confirm Bartter syndrome, the electrolyte abnormalities (childhood hypopotassemia) seems to reflect a pseudo Bartter syndrome related to undiagnosed $\mathrm{CF}$. Our patient has a less common $\mathrm{CF}$ mutation, heterozygote $2183 \mathrm{AA}>\mathrm{G}$, and a mild disease without pancreatic insufficiency. The disease condition is associated with a rare manifestation of $\mathrm{CF}$, acute pancreatitis.

\section{References}

1. J. Matt Brunson MD, Debbie Bridges MD, Robert Anderson MD Nichole Graves MD, Christopher Schwann BS. Cystic fibrosis diagnosed at age 45 based on symptoms of acute pancreatitis, Proc (Bayl Univ Med Cent).2009;22(1): 13-15.

2. Banks PA, Freeman ML. Practice guidelines in acute pancreatitis. Am Gastroenterol2006;101(10):2379-2400.

3. Pandol SJ, Saluja AK, Imrie CW, Banks PA. Acute pancreatitis: bench to the bedside. Gastroenterology2007;132(3):1127-1151.

4. Laurie Barclay MD. New Guidelines Released for Acute Pancreatitis Management 2013.

5. Singer MV, Gyr K, Sarles H. Revised classification of pancreatitis. Report of the Second International Symposium on the Classification of Pancreatitis; March28-30, 1984; Marseille, France. Gastroenterology1985;89(3):683-685.
6. Etemad B, Whitcomb DC. Chronic pancreatitis: Diagnosis, classification, and new genetic developments. Gastroenterology2007;120(3):682-707.

7. Dimagno MJ, Dimagno EP. Chronic pancreatitis. Curr Opin Gastroenterol2012;28(5):523-531.

8. Whitcomb DC. Hereditary Pancreatitis: New insights into acute and chronic pancreatitis. Gut1999; 45(3):317-322.

9. Corey Foster, Neville Mistry, Parvin F. Peddi, Shivak Sharma. Washington Manual of Medical Therapeutics. Newyork: Lippincott Williams Wilkins publishers, 2010

10. De Boeck K, Weren M, Proesmans M, Kerem E. Pancreatitis among patients with cystic fibrosis: correlation with pancreatic status and genotype. Pediatrics2005;115:463-469.

11. Walkowiak J, Lisowska A, Blaszczynski M. The changing faces of the exocrine pancreas in cystic fibrosis: pancreatic sufficiency, pancreatitis and genotype. Eur J Gastroenterol Hepatol2008;20:157-160.

12. Augarten A, Ben Tov A, Madgar I, et al. The changing face of the exocrine pancreas in cystic fibrosis: the correlation between pancreatic status, pancreatitis and cystic fibrosis genotype. Eur J Gastroenterol Hepatol2008;20:164-168

13. Ooi CY, Dorfman R, Cipolli M, Gonska T, Castellani C, Keenan K, et al. Type of CFTR mutation determines risk of pancreatitis in patients with cystic fibrosis. Gastroenterology2011;140(1):153-161.

14. Frentescu L. Mutations in the CFTR gene in patients with cystic fibrosis in Romania, Clujul Medical2010;83:163-165.

15. Leonardi S., Domenico Praticò A., La Rosa M, Early acute pancreatitis in a child with compound heterozygosis F508/R1438W/Y1032C cystic fibrosis: a case report. J Med Case Rep2013;7(1);188. 